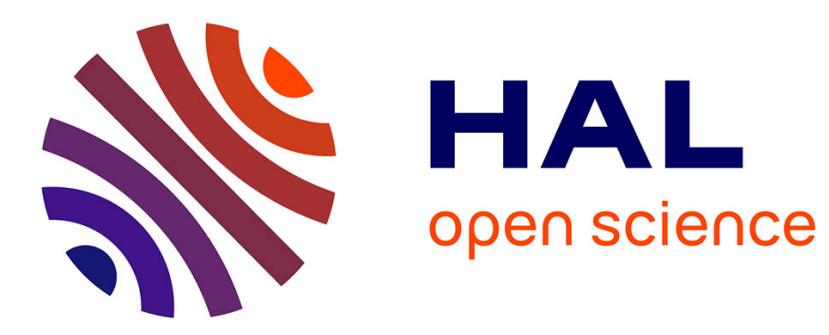

\title{
Vincent Laisney (dir.), Les souvenirs littéraires, Liège, Presses universitaires de Liège, 2017, 400 p.
} Jean-Baptiste Amadieu

\section{To cite this version:}

Jean-Baptiste Amadieu. Vincent Laisney (dir.), Les souvenirs littéraires, Liège, Presses universitaires de Liège, 2017, 400 p.. Annales. Histoire, Sciences sociales, 2018, pp.891-895. 10.1017/S0395264918000070 . hal-01971765

\section{HAL Id: hal-01971765 https://hal.science/hal-01971765}

Submitted on 7 Jan 2019

HAL is a multi-disciplinary open access archive for the deposit and dissemination of scientific research documents, whether they are published or not. The documents may come from teaching and research institutions in France or abroad, or from public or private research centers.
L'archive ouverte pluridisciplinaire HAL, est destinée au dépôt et à la diffusion de documents scientifiques de niveau recherche, publiés ou non, émanant des établissements d'enseignement et de recherche français ou étrangers, des laboratoires publics ou privés. 
Les Souvenirs littéraires, actes du colloque des 2-3-4 juin 2016 à l'université de Paris Nanterre, dir. Vincent Laisney, Liège, Presses Universitaires de Liège, coll. Situations (n $\left.{ }^{\circ} 10\right), 2017,398 \mathrm{p}$.

Vincent Laisney a organisé en juin 2016 un colloque sur les souvenirs littéraires. La publication des actes comprend, outre les contributions, l'ensemble des discussions, le procèsverbal de la table-ronde et la conférence performée d'Emmanuel Giraud. Une annexe bibliographique recense plus de trois cent titres de souvenirs littéraires, de 1830 à 2010, classés par ordre chronologique, où les chercheurs en littérature française des $\mathrm{XIX}^{\mathrm{e}}$ et $\mathrm{XX}^{\mathrm{e}}$ siècles puiseront une documentation précieuse. Les souvenirs littéraires sont en effet une mine de renseignements pour les historiens de la littérature, comme en témoignent les biographes réunis en table-ronde. V. Laisney nous confie y avoir recueilli, lui aussi, des informations utiles à ses recherches, avant de prendre conscience de la "cohérence morphologique et discursive d'un type de livre réputé pour leur caractère composite, insaisissable, évanescent » (p. 9). Le colloque s'est fixé comme objectif novateur de modéliser cette classe d'écrits souvent réduits à leur seul usage documentaire et de ce fait dévalorisés, de s'interroger sur leur genre, leur poétique et leur histoire, dont l'âge d'or se situe entre la fin du Second Empire et les Trente Glorieuses.

\section{Déterminer le genre}

Comment définir ce type de textes ? Plusieurs contributeurs avancent une définition. Jean-Pierre Bertrand en élabore une le long de son article à partir d'une analyse formelle : les auteurs sont des porte-parole, souvent des disciples, qui s'expriment sur un ton anecdotique de causerie pour soutenir une vérité ou dissiper un malentendu; derrière le chaos des cénacles, ils affirment l'influence d'individus ou d'un groupe sur l'histoire littéraire et construisent une mythologie. J.-P. Bertrand intègre ainsi à la définition « l'intention de fixer une vérité historique, de la dater et de faire date » (p. 143).

Les contributions développent les caractéristiques du genre. V. Laisney énumère le goût pour les indiscrétions sur la vie intime des célébrités, la nostalgie d'un âge d'or englouti, la peinture de portraits, le récit d'anecdotes. Pierre-Jean Dufief analyse la vocation d'écrivain racontée par les souvenirs, les stratégies mises en œuvre par les petits naturalistes pour pénétrer le milieu littéraire. La vie faite d'expédients et le vagabondage artiste sont aussi des lieux communs du genre, comme le note Anthony Glinoer : "Combien consacrent un chapitre ou quelques pages à l'arrivée à Paris, aux années d'études, aux premières amours, à la mansarde, au café, et se souviennent avec émotion de ces années de galère ? » (p. 302) Antoine Piantoni s'intéresse, en particulier, au biotope naturel de ces générations, entre Montmartre et le quartier latin, aux galeries de portraits « en action », souvent brossés sur un mode déambulatoire, et aux interruptions dans le récit des visites pittoresques pour évoquer sur un ton élégiaque les chers disparus.

Quelles sont les frontières du corpus ? À côté des stricts récits monographiques, certains contributeurs ajoutent les préfaces, les entretiens, les témoignages indirects ; Luc Fraisse y joint même, parmi les témoignages sur Proust, un roman à clés où il apparaît sous un nom d'emprunt. Certains livres de souvenirs sont des compilations d'études parues dans la presse. Aussi la frontière entre textes littéraires et articles de périodiques paraît-elle floue. Selon Julien Schuh et Yoan Vérilhac, les publications symbolistes dialoguent avec la presse et suivent un calendrier médiatique, au rythme des recensions, des nécrologies, des anniversaires, des bons mots dont sont avides les bohèmes et qui font mouche dans les journaux. Au-delà des seuls supports textuels de la mémoire, Martine Lavaud réfléchit à la 
photographie et à son rapport avec les souvenirs littéraires. Si ceux-ci s'écrivent d'abord sans (voire contre) cet art encore mal reconnu, il accompagne ensuite la remémoration et en féconde l'écriture.

Quant au corpus des seuls récits, se distingue-t-il des écrits mémoriels ? Deux contributions s'attachent à les singulariser vis-à-vis des formes proches. L'analyse discursive de Dominique Maingueneau, d'abord, les situe dans l'ensemble des memorabilia depuis l'Antiquité. À la différence des textes mémorables, le narrateur des souvenirs littéraires est présent à la fois comme énonciateur et comme acteur, il se met en scène. Tel n'est pas le cas des évangiles, où l'énonciateur et le témoin s'effacent. Si ce trait formel a le mérite de formuler une distinction claire, certains textes du corpus n'entrent pas dans cette catégorie : l'étude de cas d'Alain Pagès relève "une énonciation tendant à effacer la personnalité du témoin » (p. 24).

L'autre contribution attachée à relever la particularité des souvenirs, celle de Damien Zanone, plus resserrée sur les $\mathrm{XVIII}^{\mathrm{e}}$ et $\mathrm{XIX}^{\mathrm{e}}$ siècles, explore les ruptures et les continuités entre les souvenirs et les mémoires en partie littéraires (fréquentation des gens de lettres dans les Confessions de Rousseau, introduction de Marmontel dans le salon de Mme de Tencin). En guise de distinction entre souvenirs et mémoires, il s'appuie sur l'idée d'autonomisation de la littérature autour de 1830 : avant cette date, la vie littéraire narrée dans les mémoires paraît selon les mêmes codes que ceux employés pour décrire le reste du monde public alors que les souvenirs s'en affranchissent plus tard, d'où le passage de relai à la génération suivante et le succès du genre à partir de 1850. La distinction selon un unique critère soulève des objections. Antoine Compagnon montre que Thibaudet «ne distingue pas les deux appellations comme nous tendons à le faire en opposant les grands et la piétaille, mémoires de généraux et souvenirs de caporaux » (p. 259).

\section{Tensions dans le genre}

La plupart des contributions s'élaborent à partir d'études de cas, de la bohème à Proust, des naturalistes aux surréalistes, révélant ainsi la variété inhérente au genre et même les tensions qui le traversent. Le premier lieu de débat porte sur la valeur littéraire de cette collection de témoignages, face à laquelle la critique s'est montrée partagée. Un fort soupçon d'indignité s'est fixé sur ces textes: ils furent associés à la culture médiatique plutôt qu'artistique. Leurs auteurs sont souvent jugés comme des minores, voire des ratés, impuissants à accéder à la reconnaissance par la création et tentant de l'obtenir par l'étalage de leurs fréquentations. Par les ragots qu'ils colportent, on leur a dénié toute prétention littéraire. Enfin, l'usage documentaire semble les exclure d'une appréciation esthétique et gratuite.

Pourtant, A. Compagnon le note, Thibaudet, loin de partager un tel mépris, les rangeait parmi les écrits littéraires et les jugeait même d'un style plus authentique (cité p. 259). L'appel à communication du colloque rompt avec la dévalorisation du genre pour s'interroger sur son statut esthétique. Une contribution particulièrement symptomatique de ce nouveau regard est celle de Jean-Michel Pottier, explorant les manuscrits des souvenirs composés par Rosny aîné. Le choix d'une telle démarche, apparentée à l'édition philologique et à la génétique (souvent réservées aux textes canoniques), participe à cette réévaluation. Elle révèle en outre un souci des choix lexicaux et stylistiques, preuve d'une intention esthétique.

Un autre point épineux concerne la rhétorique épidictique des souvenirs. Le corpus comprend bon nombre d'œuvres vouées à tresser des louanges. Les témoins décrivent une «atmosphère chargée d'émotion» (A. Piantoni, p. 58) et contribuent «à la fabrique de l'écrivain de génie » (Thierry Poyet, p. 109). L'éloquence encomiastique prend un tour hagiographique en ce temps de sacralisation littéraire sous la plume des disciples, voire 
s'exaspère en une « sursacralisation » comme le montre Pascal Durand au sujet de Mallarmé. La représentation élogieuse du passé ne se limite pas à une admiration solennelle ; A. Piantoni rend compte de la célébration du pittoresque et de l'esprit de facétie chez les anciens bohèmes. Le décalage entre le temps raconté et le moment de l'écriture, s'il favorise une idéalisation du passé perdu, est cependant à double tranchant.

Certains auteurs n'hésitent pas, en effet, à prendre de la distance avec leurs enthousiasmes de jeunesse: P.-J. Dufief remarque que la «représentation sacralisée se déconstruit peu à peu sous le poids d'exigences nouvelles dont témoignent les livres de souvenirs des "petits" naturalistes qui démythifient la vocation» (p. 32). La rhétorique épidictique bascule même dans la satire (portrait de Leconte de Lisle par Albalat, cité par D. Maigueneau, p. 154-155) voire dans l'éreintement, illustré par l'étude d'A. Compagnon sur les souvenirs de Léon Daudet, dans une veine qui rappelle « la tradition haute en couleur du style pamphlétaire de droite » (p. 258). On ne saurait donc limiter le genre à des éloges de convention, d'autant que les souvenirs sont souvent moins superficiels qu'on ne l'a pensé ; ils ont aussi à cœur d'expliquer un milieu ou une personnalité. L. Fraisse étudie ainsi la manière dont les souvenirs sur Proust cherchent volontiers un fil directeur (sa conversation comme préparation de l'œuvre), enquêtent sur une source décisive à l'origine d'une scène ou d'un personnage. Au demeurant, louer et expliquer ne s'excluent pas, puisque les deux s'articulent en une même volonté de légitimer des auteurs ou une école.

Cette fonction légitimante des souvenirs littéraires soulève elle aussi des difficultés. Comme le constate Corinne Saminadayar-Perrin, « les Souvenirs de la vie littéraire justifient et légitiment des postures, des positionnements, des trajectoires » (p. 122). Ils légitiment d'abord les figures de proue. L'étude de P. Durand décèle dans l'icône que le cénacle façonne de Mallarmé, la production d'une croyance collective dans le caractère incalculable de son œuvre et de la littérature, à tel point que, plus qu'une poétique, c'est une politique qui émerge des souvenirs de disciples. Ces textes légitiment aussi ceux qui les écrivent, selon D. Maingueneau ; avoir pénétré un milieu littéraire sacré consacre à son tour le témoin, quand bien même il ne serait qu'un second couteau.

Cependant, cette fonction légitimante souffre d'ambivalence : d'abord, certains témoins ne cèdent pas à la glorification et font même montre de leur « volonté de livrer un véritable portrait, s'opposant [...] aux légendes simplificatrices » (A. Pagès, p. 24); ensuite, le bilan est aussi l'occasion d'un mea culpa (P.-J. Dufief rapporte les remords du Manifeste des Cinq, p. 37) ; enfin, les laissés pour compte de la notoriété prennent leur revanche sur les grands, ainsi que l'explique V. Laisney. Ces ombres portées à la consécration érodent-elles l'image des gloires littéraires ? Selon Thibaudet, même le fiel, en montrant les ridicules des écrivains de renom, les rend «paradoxalement plus humains » (p. 261).

Qu'ils en donnent une image avantageuse ou ingrate, les souvenirs ont-ils seulement vocation à traiter du passé ? On pourrait les croire seulement tournés vers un monde révolu. C. Saminadayar-Perrin étudie les griefs que Vallès adressa aux récits nostalgiques de la vie de bohème; le romancier dénonce leurs illusions désuètes et leur valeur de modèle qui conduit les artistes à la misère. Maxime Du Camp et Ernest Feydeau ne parviennent pas à sortir des regrets pour se tourner vers l'avenir, à passer de l'anecdotique à l'essentiel et à s'élever audessus du moi ; ils échouent ainsi à s'imposer comme de vrais littérateurs dotés d'une position et d'un rayonnement, selon T. Poyet.

D'autres souvenirs, pourtant, ne s'arrêtent pas à cette orientation rétrospective, en particulier pour les textes relatifs à la bohème ou au surréalisme, pour lesquels l'histoire est constitutive du mouvement. Selon A. Glinoer, les œuvres de la bohème revivent par le discours les scènes théâtralisées de sa geste. Elle s'autoproduit par ses propres récits ; son chroniqueur lui-même, Murger, devient à son tour objet de récits. Quant au surréalisme, il est constitué par la narration des événements fondateurs. L'élaboration en continu du récit 
mémorable organise la disparate ; la légende officielle stabilise le mouvement, par exemple en circonscrivant dada à un moment négatif d'une dialectique historique, comme l'explique Michel Murat. Cette narration constitutive du mouvement reste traversée de tensions : si Breton et Aragon ont tendance à tempérer les différends (et Aragon à combler le hiatus de sa trajectoire), Soupault règle ses comptes avec le despotisme de Breton, de sorte que la mémoire est tiraillée, « opérant par grossissement et atténuation » (p. 253), selon l'expression de David Vrydaghs.

L'enjeu historiographique des souvenirs est doublement polémique : d'une part en raison des divergences de vue entre acteurs, d'autre part parce que ces textes composent une histoire littéraire d'écrivains sans la distance profitable à l'histoire savante. Les contributeurs soulignent les soupçons qui pèsent sur la véracité de ces témoignages : failles de la mémoire, épisodes connus par ouï-dire, mauvaise foi, plaidoyer pro domo, dilemme entre exactitude et efficacité du récit (pas toujours au bénéfice de la première), construction d'une légende. La perplexité académique affecte l'authenticité des faits relatés, mais encore les considérations de critique littéraire auxquelles s'adonnent de tels témoins, plus mondains qu'érudits. L. Fraisse fait la part des choses entre les mérites des uns et des autres : si les auteurs de souvenirs ont conscience « de ne pas être des analystes professionnels des œuvres dont ils ont personnellement approché les auteurs », ils furent «placés en situation de témoins oculaires qu'aucun professionnel de la postérité ne pourra occuper » (p. 182).

Le dépoussiérage entrepris par ce colloque peut servir d'aiguillon à divers développements. Deux extensions me paraissent envisageables. 1) En prolongement des comparaisons avec les écrits mémoriels situés en amont de la période, un travail qui considérerait la littérature testimoniale après 1945 serait bienvenu. Les études autour d'Antelme par exemple, sur la transmission d'une vérité factuelle par des procédés littéraires, interrogeraient l'amalgame du littéraire au factice et la présomption d'insincérité à laquelle sont parfois exposés les souvenirs. 2) Plusieurs auteurs ont analysé le corpus à la lumière des concepts de sacralisation, d'autonomisation et de légitimation. La démarche inverse, en examinant à nouveaux frais la validité de ces catégories à l'aide des témoignages, s'avérerait également opportune. Espérons que ce colloque pionnier soit le premier jalon d'études sur un genre à redécouvrir. 